\author{
Radu Ardevan \\ (Cluj-Napoca)
}

\title{
ÜBER DAS RELIGIÖSE VERHALTEN DER MUNIZIPALARISTOKRATIE VON SARMIZEGETUSA*
}

\begin{abstract}
One can research the religious life and choices of the local aristocrats through the analysis of their private dedications only. The results prove that these people were personally less involved in the imperial cult or in the trendy exotic religions, preferring the Roman deities, mainly Jupiter and the ones defending the home, the welfare and the health.
\end{abstract}

\section{Key words}

Sarmizegetusa, municipal aristocracy, religion, cults, gods, dedications

* Eine erste Version dieser Arbeit wurde als Poster im Rahmen des XIV. Internationalen Kongresses für griechische und lateinische Epigraphik (Berlin, 27.-31. August 2012) präsentiert. Inzwischen hat die Dokumentation zugenommen, und unsere Ansicht über das Forschungsthema wurde vertieft. Unsere Forschung wurde durch Mittel der Nationalen Rumänischen Behörde für Wissenschaftliche Forschung, CNCS-UEFISCDI, Projektnummer PN-II-ID-PCE-2011-3-0096 unterstützt. 
Die Kolonie Ulpia Traiana Sarmizegetusa ist die älteste und die wichtigste Stadt des römischen Dakiens ${ }^{1}$. Auch wenn sie nie als Provinzhauptstadt fungierte ${ }^{2}$, verfügte sie über ein ausgedehntes Territorium ${ }^{3}$ und erfreute sich eines hohen Ansehens, wie eine echte colonia deducta mit einer großen Anzahl alter römischer Bürger ${ }^{4}$, sowie als der erste Ort wo der Jupiter- und Kaiserkult eingeführt und praktiziert wurden ${ }^{5}$, aber auch als Mutterstadt (Metropolis) für manche andere Städte ${ }^{6}$. Ihre Munizipalaristokratie (ordo decurionum) ist die reichste und am besten vertretene aus der ganzen Provinz. Um die 260 Inschriften dokumentieren diese Sozialschicht ${ }^{7}$.

Es ist aber viel schwieriger das religiöse Verhalten dieser Elite zu kennen. In diesem Sinne können nur ihre Votivinschriften als Quellen dienen. Eine solche Untersuchung ist für die historische Kenntnis von Interesse, denn sie kann manche Elemente der Geistigkeit der Gemeinde und der Kollektividentität erhellen.

Dafür braucht man erstens die zur Verfügung stehenden Quellen auswählen. Es wird aber notwendig, eine neue Überprüfung der den Glauben der ulpianischen Elite widerspiegelnden Inschriften vorzunehmen. Von Anfang an werden die Denkmäler der hohen kaiserlichen Beamten und des Militärs ausgeschlossen, weil diese nicht als Teil der örtlichen Gesellschaft zu

${ }^{1}$ I. Piso, An der Nordgrenze des Römischen Reiches. Ausgewählte Studien (1972-2003), Stuttgart 2005, S. 437; idem (Hg.), Le forum vetus de Sarmizegetusa, I, Bucureşti 2006, S. 70-72; R. Ardevan, Colonia Ulpia Traiana Sarmizegetusa: les institutions politiques et religieuses, [in:] idem, R. Varga (Hg.), Trajan und seine Städte. Colloquium Cluj-Napoca, 29. September-2. Oktober 2013, Cluj-Napoca 2014, S. 275-276.

${ }^{2}$ R. Haensch, Capita provinciarum. Statthaltersitze und Provinzialverwaltung in der römischen Kaiserzeit, Mainz 1993, S. 338-348; I. Piso, Fasti provinciae Daciae II. Die ritterlichen Amtsträger, Bonn 2013, S. 6.

${ }^{3}$ I. Piso, An der Nordgrenze, S. 273-293; R. Ardevan, Viaţa municipală în Dacia romană, Timişoara 1998, S. 74-81, 112-113.

${ }^{4}$ I. Piso, Die soziale und ethnische Zusammensetzung der Bevölkerung in Sarmizegetusa und in Apulum, [in:] W. Eck (Hg.), Prosopographie und Sozialgeschichte. Studien zur Metodik und Erkentnismöglichkeit der kaiserzeitlichen Prosopographie (Kolloquium Köln, 24.-26. November 1991), Köln-Wien-Weimar, 1993, S. 321-324; R. Ardevan, Viaţa municipală, S. 276.

${ }^{5}$ R. Etienne, I. Piso, Al. Diaconescu, Les deux forums de la Colonia Ulpia Traiana Augusta Dacica Sarmizegetusa, Revue des Etudes Anciennes 92, 1990, 3-4, S. 279-280; I. Piso, An der Nordgrenze, S. 186-187, 442, 448-449; I. Piso, Il Capitolium, l'Epulum Iovis e il Dies Iovis nella Dacia romana, [in:] L. Zerbini (Hg.), Roma e le province del Danubio. Atti del I Convegno Internazionale Ferrara - Cento, 15-17 Ottobre 2009, Soveria Manelli 2010, S. 273; I. Piso, F. Marcu, G. Cupcea, O. Țentea, R. Varga, Das Kapitol von Sarmizegetusa, Dacia. Revue d'archéologie et d'histoire ancienne. Nouvelle série 56, 2012, S. 120-121; R. Ardevan, Colonia Ulpia Traiana Sarmizegetusa, S. 276, 280.

${ }^{6}$ R. Ardevan, Viața municipală, S. 113-117; I. Piso, An der Nordgrenze, S. 449.

${ }^{7}$ R. Ardevan, Viaţa municipală, S. 188-208; ILS 190, 247, 248, 288, 554 (?), 862, 890, 891. 
betrachten sind ${ }^{8}$. Es wurden ebenfalls auch die Inschriften der Augustalen weggelassen, da sie keine echte Munizipalaristokraten sind ${ }^{9}$. Dasselbe gilt auch für die Votivdenkmäler der Dekurionen anderer Städte ${ }^{10}$, eben wenn sie als sacerdotes arae Augusti agiert haben ${ }^{11}$. Wir haben ebenfalls einige Inschriften von Personen ausgeschlossen, die nach den letzten Forschungen nicht der Aristokratie von Sarmizegetusa einzugliedern sind ${ }^{12}$, oder kaum zu dieser Sozialschicht zu rechnen sind ${ }^{13}$. Gemieden wurden auch einige Stücke, deren Votivcharakter umstritten bleibt ${ }^{14}$.

Es bleiben also 65 nützliche Inschriften für die vorliegende Untersuchung, ungefähr $25 \%$ von dem oben Eingeschätzten. Die zahlreichsten stammen gerade aus Sarmizegetusa (29 Stücke, 45\%), es kommen aber auch außerhalb dieser, aus ihrem Territorium vor $(20 \text {, d.h. } 30 \%)^{15}$ oder sogar von jenseits (16 Denkmäler, 24\% $)^{16}$. Ihre Zerstreuung widerspiegelt ziemlich gut die Anwesenheit der ulpianischen Aristokratie im Leben der Provinz.

Diese epigraphischen Quellen können ihrerseits in zwei große Kategorien eingetelit werden. Die erste, bei weitem die zahlreichste, besteht aus den

${ }^{8}$ R. Ardevan, Viaţa municipală, S. 178. Eine Votivstatue wurde von einem anonymen Ritter in dem Forum aufgestellt, es ist aber nicht sicher, ob der Widmer irgendeine Würde in Sarmizegetusa bekleidet hat (I. Piso, Forum vetus, CEp 71).

${ }^{9}$ R. Duthoy, Les Augustales, ANRW II 16/2, 1978, S. 1281-1285; R. Ardevan, Viaţa municipală, S. 243-245; R. Ardevan, Colonia Ulpia Traiana Sarmizegetusa, S. 282.

${ }^{10}$ Z. B. AE 2004, 1211 = ILD 881.

${ }^{11}$ Es ist der Fall von P. Aelius Antipater (IDR III/2, 217), M. Antonius Valentinus (CIL III 1433 = IDR III/2, 266) oder Ti. Claudius Augustianus (AE 1903, 69 = IDR III/2, 79; IDR III/5, 394).

${ }^{12}$ Den Dekurionen C. Aelius Primus (CIL III 7788 = IDR III/4, 32; CIL III 1030 = IDR III/5, 106) haben wir zu erst als ulpianer betrachtet (R. Ardevan, Viaţa municipală, S. 194), aber die neuren Forschungen neigen dazu, ihn als einen von Apulum zu sehen (IDR III/5, 106, 128).

${ }^{13}$ Das Dekurionenamt ist für P. Aelius Artemidorus, sacerdos des Mithras (CIL III $12555=$ IDR III/4, 30), äußerst hypothetisch. Was die Inschrift CIL III 1333 = IDR III/3, 344 von Ampelum anbelangt, statt [Ul]p. Maxim[us / et Ul]p. Avi[tus? / IIv(iri?)] i. d., ziehen wir die Lesung [Ul]p. Maxim[us / ....p]r. aur[a]r[iar(um)] / ... IDV ... vor (H.-Chr. Noeske, Studien zur Verwaltung und Bevölkerung der dakischen Goldbergwerke in römischer Zeit, Bonner Jahrbücher des Rheinischen Landesmuseums 177, 1977, S. 356, AMP 24), also bezieht sich das nicht auf Würdenträger aus Sarmizegetusa (R. Ardevan, Viaţa municipală, S. 419, R 387). Die Widmung von Domnești (CIL III 7625 = ILD 804 = ILD 1203), obwohl zur Gesundheit eines Dekurionen gedacht, drückt eigentlich die Pietät seines Sklaven aus.

${ }^{14}$ Trotz des scheinbaren Aussehens, sind die Stücke CIL III $1412=7902$ und AE 1972, 463 Honorarinschriften. Die Stücke AE 2003, 1517 = I. Piso, Forum vetus, CEp 10 und AE 1912, $76=$ IDR III/2, $6=$ AE 2003, 1518 beziehen sich auf den Bau eines Gebäudes (I. Piso, Forum vetus, CEp 11).

${ }^{15}$ Für die Entwicklung des Stadtterritoriums vgl. I. Piso, An der Nordgrenze, S. 273-293 und R. Ardevan, Viaţa municipală, S. 74-81, 112-113.

${ }^{16}$ Nur eine einzige Inschrift dieser Art erscheint außerhalb Dakiens, in Mytilenae (siehe IGR IV $/ 1,47=$ Dobo $^{4} 849=$ IDRE II, 372). 
persönlichen Widmungen der Aristokraten von Sarmizegetusa. Viel weniger sind die Texte, die Widmungen der Stadt selbst, also Urkunden einer offiziellen Frömmigkeit, darstellen - bloß fünf an der Zahl ${ }^{17}$. Unter den letztgenannten verzeichnet eine das örtliche Jupiter-Fest und zugleich die Einweihung des Stadtkapitols ${ }^{18}$; die anderen vier erinnern an religiöse Zeremonien der Stadt, zur Unterstützung des Kaisers, an die traditionellen römischen Götter ${ }^{19}$ oder an exotische in die römische Welt eingegliederte Gottheiten (nämlich Isis und Serapis) ${ }^{20}$.

Die übrigen 60 persönlichen Widmungen der ulpianischen Aristokraten widerspiegeln umfassend ihre religiöse Geisteswelt und die forma mentis dieser Elite. Die von ihnen verehrten Gottheiten können in sechs Kategorien eingeteilt werden, wobei jede ihr spezifisches Gewicht in den Quellen und in dem Leben der Gläubigen hat. Wir werden sie der Reihe nach behandeln.

\section{JUPITER OPTIMUS MAXIMUS}

Abgesehen von der Anwesenheit des römischen Hauptgottes in den obengenannten offiziellen Urkunden ${ }^{21}$, verfügen wir nur über 10 epigraphische Denkmäler, die von Privatpersonen den Jupiter gewidmet wurden $(15,38 \%$ aus der Gesamtzahl). Es ist weniger als wir vermutet haben, besonders wenn man an die Wichtigkeit dieses Kultes in der damaligen Zeit denkt ${ }^{22}$. Erwähnenswert ist die Tatsache, daß solche Widmungen von außerhalb von Sarmizegetusa stammen ${ }^{23}$.

Der Gott erscheint am meisten in der kanonischen Form von Iupiter Optimus Maximus. Nur in zwei Fällen bekommt er regionale Epitheta, und

${ }^{17}$ Siehe: CIL III 7958 + 7920 = IDR III/2, 227; IDR II/2, 241; IDR III/2, 242; CIL III 7986 = IDR III/2, 352; I. Piso, Forum vetus, CEp 12.

${ }^{18}$ IDR III/2, 242. Vgl. I. Piso, Il Capitolium, S. 273-277.

${ }^{19}$ IDR II/2, 241 (zu ihre Datierung und Zuschreibung - Stadtbehörde oder Provinziallandtag - siehe I. Piso, An der Nordgrenze, S. 48-50); CIL III 7986 = IDR III/2, 352.

${ }^{20}$ CIL III $7958+7920=$ IDR III/2, 227. Für ein anderen Stück (I. Piso, Forum vetus, CEp 12) bleibt die verehrte Gottheit unbekannt.

${ }^{21} \mathrm{Vgl}$. oben, Anm. 18-19.

${ }^{22}$ M. Bărbulescu, Interferențe spirituale în Dacia romană ${ }^{2}$, Cluj-Napoca 2003, S. 177; M. Popescu, La religion dans l'armée romaine de Dacie, Bucarest 2004, S. 39-49.

${ }^{23}$ Bloß je eine von Uroi (CIL III 7881 = IDR III/3, 209) und Germisara (AE 1971, 403 = IDR III/3, 236), zwei von Apulum (CIL III 1051 = IDR III/5, 164; CIL III 1060 = IDR III/5, 183), aber je drei von Aquae (CIL III 1407 = IDR III/3, 10; CIL III 12576 = IDR III/3, 17; CIL III $12575=$ IDR III/3, 18) und Ampelum (CIL III 1282, 1283 = IDR III/3, 308, 309; CIL III 942 = IDR III/3, 317; AE 1988, $955=$ ILD 334). 
zwar Appeninus ${ }^{24}$ bzw. Depulsor ${ }^{25}$ - allebeide in Aquae. Gewöhnlich kommt er alleine in den Inschriften vor; nur einmal wird er mit dem Genius imperatorum ${ }^{26}$ und zweimal mit „andern Göttern”27 in Verbindung gebracht.

Interessant scheint uns der Grund für die Ansprache Gottes. Ein einziges Mal wird der göttliche Schutz für den Kaiser ${ }^{28}$ und ein anderes Mal für einen legatus Augusti ${ }^{29}$ in Anspruch genommen. Solche Gesten könnten als einfache „Dienstpflichten” oder „Sozialverpflichtungen” angedeutet werden. Zweimal sind die Widmungen dafür errichtet, weil Jupiter auf die Bitte pro salute geantwortet hat ${ }^{30}$. In den anderen Fällen begegnen wir der kurzen Erwähnung votum solvit libens merito, was auf persönliche Gründe hinweist. Infolgedessen stellen wir die vorwiegende Anwesenheit der Privatanliegen fest. Unserer Meinung nach beweisen die uns zur Verfügung stehenden Texte eine echte Frömmigkeit.

\section{GRIECHISCH-RÖMISCHE GOTTHEITEN}

Es ist die am besten vertretene Kategorie, sie umfasst 29 Inschriften (44,6\% der Gesamtzahl), die sich an 12 verschiedene Kulte wenden ${ }^{31}$. Diese sind aber selbstverständlich sehr unterschiedlich vertreten.

Unter diesen Gottheiten scheint Silvanus für die ulpianische Elite die beliebteste zu sein; dieser Gott wird häufig in den Provinzen der mittleren Donau als Schützer des Hauses und des Haushaltes verehrt ${ }^{32}$. Für ihn sind sieben Votivstücke bekannt ${ }^{33}$. Zweimal erscheint er mit dem Epitheton

\footnotetext{
${ }^{24}$ CIL III $12576=$ IDR III/3, 17.

${ }^{25}$ CIL III 12575 = IDR III/3, 18. Iupiter Depulsor erscheint als eine für die Provinz Noricum spezifische Gottheit (M. Šašel-Kos, Pre-Roman Divinities of the Eastern Alps and Adriatic, Ljubljana 1999, S. 125-129).

${ }^{26}$ CIL III $1407=$ IDR III/3, 10.

${ }^{27}$ CIL III 942 = IDR III/3, 317; CIL III 1060 = IDR III/5, 183.

${ }^{28}$ CIL III $1407=$ IDR III $/ 3,10$.

${ }^{29}$ AE 1971, 403 = IDR III/3, 236.

${ }^{30}$ Es geht um seine eigene Gesundheit (AE 1988, 955 = ILD 334) oder die der ganzen Familie (CIL III $1060=$ IDR III/5, 183).

${ }^{31}$ Für die allgemeine Lage dieser Kulten in der Provinz siehe M. Bărbulescu, Interferențe, S. 176. Ihr Gewicht in Sarmizegetusa ist damit nicht einstimmig.

${ }^{32}$ A. Mócsy, Pannonia and Upper Moesia, London-Boston, 1974, S. 250-252; M. Bărbulescu, Interferențe, S. 222, 229-230; D. Dzino, The cult of Silvanus: rethinking provincial identities in Roman Dalmatia, Vjesnik Arheološkog muzeja u Zagrebu 3, XLV, 2012, S. 263.

${ }^{33}$ CIL III 12578 = AE 1977, 692 = IDR III/2, 332; IDR III/2, 333; CIL III 1441 = IDR III/2, 334; AE 1934, 13 = IDR III/2, 335; AE 1986, 607 = IDR III/5, 325; CIL III 1141 = IDR III/5, 330; CIL III $1152=$ IDR III $/ 5,344$.
} 
Domesticus $^{34}$, ein anderes Mal wird er bildlich mit den neun Silvanae dargestellt ${ }^{35}$; auf einen anderen Denkmal erscheint er in Verbindung mit Terra Mater und Herkules ${ }^{36}$. Die meisten Widmungen sind Folge eines votum, nur in zwei Fällen wird klar der Zweck ausgedrückt - pro salute ${ }^{37}$.

In unmittelbarer Nähe stehen Aesculapius und Hygeia, mit sechs epigraphischen Denkmälern ${ }^{38}$. Nur zweimal erscheinen diese Gottheiten getrennt ${ }^{39}$. Die Widmungen sind vor allem ex voto, nur einmal pro salute ${ }^{40}$. In Sarmizegetusa fungierte ein Asklepieion ${ }^{41}$. Nur einmal trägt der Gott das Epitheton Pergamenus ${ }^{42}$, mit direktem Bezug auf das weltberühmte Heiligtum in Pergamon ${ }^{43}$.

Der Kult der Nymphen, Gottheiten des Wassers und der Heilung ${ }^{44}$, erscheint auf vier beschrifteten Denkmälern. Ein solches Stück wurde in einem Thermalbad von den fünf Vertretern der Kolonie bei der Heimkehr errichtet ${ }^{45}$; obwohl sie sich im offiziellen Dienst befanden, verrät ihre Geste eine aufrichtige und überzeugend motivierte Pietät. Eine andere, in Germisara pro filiis errichtete Inschrift, bedeutet dasselbe ${ }^{46}$. Anders sind die von einem Würdenträger im Forum in honorem domus divinae erbaute nymphaea zu deuten; seine Evergesie drückt vor allem seine politische Treue gegenüber der neuen Dynastie aus ${ }^{47}$.

Für Nemesis, die Göttin der Rache und der Gerechtigkeit, können wir drei Inschriften erwähnen. Zwei von ihnen verzeichnen die Widmungsgeste auf

\footnotetext{
${ }^{34}$ CIL III $12578=$ AE 1977, 692 = IDR III/2, 332; AE 1934, 13 = IDR III/2, 335.

${ }^{35}$ AE $1934,13=$ IDR III/2, 335.

${ }^{36}$ CIL III $1152=$ IDR III/ $/ 5,344$.

${ }^{37}$ CIL III $12578=$ AE 1977, $692=$ IDR III/2, 332; CIL III $1152=$ IDR III/5, 344.

${ }^{38}$ CIL III 7896 = IDR III/2, 152; IDR III/2, 153; CIL III 1417a = IDR III/2, 164; CIL III 973 = IDR III/5, 316, 164; CIL III 1279, 1280 = IDR III/3, 286; CIL III 972 = IDR III/5, 1.

${ }^{39}$ CIL III $7896=$ IDR III/2, 152 (Inschrift für beide, aber auf der Basis einer Hygeiastatue); CIL III 972 = IDR III/5, 1 (numini Aesculapii).

${ }^{40}$ IDR III/2, 153.

${ }^{41}$ A. Rusu-Pescaru, D. Alicu, Templele romane din Dacia (I), Deva 2000, S. 32-38. Ein Dekurione ist sogar sacerdos des Gottes (CIL III 973 = IDR III/5, 316).

${ }^{42}$ CIL III $1417 \mathrm{a}=$ IDR III/2, 164.

${ }^{43}$ J.R. Carbó Garcia, Los cultos orientales en la Dacia romana. Formas de diffusion, integración y control social e ideológico, Salamanca 2010, S. 307-308. S. 140.

${ }^{44}$ I. Nemeti, Collective feminine goddesses in Roman Dacia, Acta Musei Napocensis 36/I, 1999,

${ }^{45}$ CIL III 1562 = IDR III/1, 56. Vgl. R. Ardevan, Viaţa municipală, S. 155-156 und R. Ardevan, Colonia Ulpia Traiana Sarmizegetusa, S. 279-280.

${ }^{46}$ AE $1992,1486=$ ILD 328.

${ }^{47}$ IDR III/2, 22 = AE 2003, 1520; AE 2003, 1521; vgl. I. Piso, Forum vetus, S. 244-248.
} 
Bilddenkmäler dieser Göttin ${ }^{48}$. Eine dritte Inschrift spricht über den Bau eines Tempels für Dea Regina ${ }^{49}$; obwohl unter diesen Namen auch andere Gottheiten (wie z. B. Juno oder Isis) stecken können ${ }^{50}$, sind wir diesmal geneigt, ebenfalls die Nemesis zu sehen ${ }^{51}$. All diese Tätigkeiten gehen auf persönliche Gründe zurück.

Es wird noch Diana mit zwei Widmungen hervorgehoben. Wenn die eine als eine persönliche Pietät $\mathrm{zu}$ deuten ist $^{52}$, steckt hinten der zweiten wahrscheinlich ein Ausdruck des politischen Konformismus, solange die Göttin auch Augusta benannt ist ${ }^{53}$. Terra Mater erhält ebenfalls zwei Widmungen, eine alleine, die andere in Verbindung mit Herkules und Silvanus ${ }^{54}$. Herkules erscheint zweimal, aber jedesmal in Verbindung mit anderen Göttern ${ }^{55}$.

Für andere Gottheiten gibt es zurzeit nur je ein Denkmal, so im Falle von Apollo ${ }^{56}$, Mars $^{57}$, Saturnus ${ }^{58}$ und Genius collegii fabrum ${ }^{59}$. Die Lage von Liber Pater, ein Gott der im mittleren Donauraum sehr beliebt ist ${ }^{60}$, ist gewissermaßen unterschiedlich: eine Inschrift erinnert an den Wiederaufbau seines Tempels von einem Dekurionen infolge eines sozialen Aufstiegs ${ }^{61}$.

${ }^{48}$ CIL III 13783 = IDR III/2, 318; CIL III 13781 = IDR III/2, 320.

${ }^{49}$ CIL III 7907 = IDR III/2, 19. Der Bau entsteht für die Gesundheit eines Verwandten.

${ }^{50}$ J. R. Carbó Garcia, Los cultos orientales, S. 379-380, 391-392.

${ }^{51}$ In Sarmizegetusa trägt sie auch das Epitheton Regina (IDR III/2, 309, 313, 314, 321), und in der Stadt gibt es ein Nemeseum (A. Rusu-Pescaru, D. Alicu, Templele, S. 61-65). Dagegen J. R. Carbó Garcia, Los cultos orientales, S. 403.

${ }^{52}$ CIL III $1281=$ IDR III/3, 293.

${ }^{53}$ CIL III $7742=$ IDR III/5, 60. Der gleiche Mensch erscheint in IDR III/2, 113 als Veteran der legio XIII Gemina Severiana.

${ }^{54}$ CIL III 1284, 1285 = IDR III/3, 330, 331; CIL III 1152 = IDR III/5, 344. Vgl. oben, Anm. 35.

${ }^{55}$ Mit Silvanus und Terra Mater (CIL III 1152 = IDR III/5, 344), bzw. mit Obila (CIL III 6263 $=$ IDR III $/ 4,29$ ).

${ }^{56}$ AE 2011, $1094=$ ILD 862.

${ }^{57}$ CIL III 12577 = IDR III/3, 19. Wahrscheinlich die gleiche Person, namens L. Grattius Paternus, wie in CIL III 1417a = IDR III/2, 164 und CIL III $12575=$ IDR III/3, 18.

${ }^{58}$ CIL III $7838=$ IDR III/3, 322.

${ }^{59}$ CIL III $1424=$ AE 1903, 64 = IDR III/2, 214. Vgl. I. Piso, Forum vetus, CEp 23.

${ }^{60} \mathrm{~A}$. Bruhl, Liber Pater. Origine et expansion du culte dionysiaque à Rome et dans le monde romain, Paris 1953, S. 78-79; C. Popescu, M. Popescu, Le culte de Liber Pater en Dacie romaine, Thraco-Dacica 16, 1995, S. 227-235; S. Pilipović, Il culto di Liber a Taurunum; un contributo allo studio della viticoltura nella Pannonia Inferiore, Eirene. Studia Graeca et Latina 52, 2016, S. 457.

${ }^{61}$ AE 1976, 561 = IDR III/2, 11; vgl. R. Ardevan, Viaţa municipală, S. 206. Für den Tempel siehe A. Rusu-Pescaru, D. Alicu, Templele, S. 52-58. 
Juno, Minerva, Salus populi Romani Quiritium, Mars Gradivus und Fortuna Redux erscheinen ein einziges Mal (zusammen mit Jupiter), in dem Text einer votorum nuncupatio ${ }^{62}$, also in einer offiziellen Akte.

Die Vorliebe der Aristokraten von Sarmizegetusa für die Gottheiten der Heilung (Aesculapius und Hygeia, Nymphen), des Hauses (Silvanus, Liber Pater, Terra Mater) und der Verteidigung (Mars, Hercules, Nemesis) ist ersichtlich. Gleichfalls ist die Hauptrolle des ehrlichen Glaubens erkennbar ${ }^{63}$. Nur in zwei (evtl. drei) Fällen gibt es Widmungen mit Anspielungen an das kaiserliche Haus ${ }^{64}$.

Es ist noch $\mathrm{zu}$ bemerken, daß diese Sozialschicht meistens einfache Votivstücke zur Schau stellt; nur dreimal erfahren wir über Bautätigkeiten mancher Euergeten für Tempel ${ }^{65}$, zu dem noch der Bau von zwei öffentlichen Fontänen kommt ${ }^{66}$.

Ein bezeichnendes Detail ist, daß zwei Mitglieder der ulpianischen Aristokratie religiöse Würden Laurentes Lavinates in Italien bekleidet haben ${ }^{67}$; obwohl diese keine Ortsämter bilden,weisen sie auf die Einschätzung der Gesellschaft in Sarmizegetusa gegenüber den ältesten religiösen römischen Traditionen ${ }^{68}$.

\section{GOTTHEITEN AUS DEM ORIENT}

Es gibt zwölf solchen Gottheiten gewidmeten Inschriften (18,46\%), die neun unterschiedliche Götter nennen. Man soll aber die exotischen Kulte Orients von den ebenfalls orientalischen Religionen, die aber zur Zeit der

\footnotetext{
${ }^{62}$ IDR II/2, 241. Siehe oben, Anm. 19.

${ }^{63}$ Bloß in zwei Fällen kann man die Votivgesten mit vermutlichen „Dienstpflichten” gleichsetzen (ein Widmer war sacerdos des Gottes, AE 2011, $1094=$ ILD 862; ein anderer wird eine Rolle im collegium fabrum gespielt haben, CIL III $1424=$ AE 1903, $64=$ IDR III/2, 214, vgl. I. Piso, Forum vetus, CEp 23), in anderen vier Fällen darf man einen persönlichen Ehrgeiz zum Sozialaufstieg vermuten (AE 1976, 561 = IDR III/2, 11; IDR III/2, $22=$ AE 2003, $1520=$ I. Piso, Forum vetus, CEp 25; AE 2003, 1521 = I. Piso, Forum vetus, CEp 26; CIL III 1417a = IDR III/2, 164).

${ }^{64}$ IDR III/2, 22 = AE 2003, 1520; AE 2003, 1521 (für beide vgl. I. Piso, Forum vetus, S. 244-248); vielleicht auch CIL III 7742 = IDR III/5, 60

${ }^{65} \mathrm{AE}$ 1976, 561 = IDR III/2, 11; CIL III 7907 = IDR III/2, 19; CIL III 1417a = IDR III/2, 164.

${ }^{66}$ Siehe oben, Anm. 63.

${ }^{67}$ CIL III 6270 = IDR III/2, 2; CIL III $1509=$ IDR III/2, 3; CIL III $1198=$ AE 1993, $1333=$ IDR III/5, 598. Vgl. I. Piso, Forum vetus, S. 258-262. S. 281.

${ }^{68}$ R. Ardevan, Viaţa municipală, S. 235-239; R. Ardevan, Colonia Ulpia Traiana Sarmizegetusa,
} 
Provinz Dakien seit langem in die römische Gesellschaft eingeführt wurden, trennen. Wir beziehen uns zuerst auf diese zweite Kategorie.

Cybele, als Magna Deum Mater verzeichnet ${ }^{69}$, erscheint ein einziges Mal. Ein in der ulpianischen Aristokratie eingedrungener Veterane, zusammen mit seiner Familie, errichtet dieser Gottheit ein Votivdenkmal pro salute August; es geht hier um eine Widmung mit sozial-politischer konformistischer Absicht ${ }^{70}$. Jupiter Dolichenus ${ }^{71}$ wird ebenfalls ein einziges Mal verehrt, ohne ausdrückliche Bestätigung, durch eine spezifische Skulptur $^{72}$. Dasselbe gilt auch für Serapis - eine Widmung zu seinem numen $^{73}$. Dagegen verfügen wir für den Gott Mithras über zwei Denkmäler: eine Votivplatte Invicto ... pro salute gewidmet ${ }^{74}$ und eine aus einem Heiligtum stammende Votivsäule ohne ausdrückliche Erwähnung der Gottheit ${ }^{75}$.

Weitere fünf Inschriften erwähnen andere drei vom Orient abstammenden Götter, die aber im 2.-3. Jh. n. Chr. in Mode waren und Anhänger aus dem römischen Milieu erworben hatten. Drei Widmungen werden dem Gott Aeternus ${ }^{76}$, alle aus persönlichen Gründen angeboten ${ }^{77}$. Genauso sieht es auch mit dem Altar für die synkretische Gottheit Theos Hypsistos ${ }^{78}$ aus, die in der griechischen ägeischen Umwelt in Mytilenae erscheint ${ }^{79}$. Nur das einzige Denkmal für Bonus Puer ${ }^{80}$ wurde von dem Widmer $o b$ honorem

\footnotetext{
${ }^{69}$ J.R. Carbó Garcia, Los cultos orientales, S. 301-304.

${ }^{70}$ CIL III $1100=$ IDR III/5, 253.

${ }^{71}$ Er ist ein wichtiger und viel verehrter Gott in römischen Dakien (M. Popescu, La religion, S. 135; J.R. Carbó Garcia, Los cultos orientales, S. 187).

${ }^{72}$ IDR III/2, 201.

${ }^{73}$ CIL III 973 = IDR III/5, 316 (neben dem oben genannten offiziellen Text für Isis und Serapis von CIL III $7958+7920=$ IDR III/2, 227 - siehe Anm. 20).

${ }^{74}$ CIL III 7959 = IDR III/2, 226. Zum Problem des Epithetons Invictus, vgl. J. R. Carbó Garcia, Los cultos orientales, S. 392, 433-450.

${ }^{75}$ CIL III 7941 = IDR III/2, 293 (eine von zwei Munizipaldekurionen ex voto errichtete Säule).

${ }^{76}$ Vgl. J. R. Carbó Garcia, Los cultos orientales, S. 192-193.

${ }^{77}$ CIL III 7996 = IDR III/1, 133 = AE 1997, 1297; IDR III/3, 215; AE 1947, 22 = IDR III/5, 29. Vgl. R. Ardevan, Viața municipală, S. 136.

${ }^{78}$ S. Sanie, Cultele orientale în Dacia romană. I Cultele siriene şi palmiriene, Bucureşti 1981, S. 156-163; J.R. Carbó Garcia, Los cultos orientales, S. 194.

${ }^{79}$ IGR IV/1, $47=$ Dobo $^{4} 849=$ IDRE II, 372. Für die Herkunft des Widmers siehe: R. Ardevan, Viaţa municipală, S. 195-196, bzw. S. Nemeti, I. Nemeti, Theos Hypsistos epekoos la Sarmizegetusa, [in:] C. Gaiu, Cr. Găzdac (Hg.), Fontes historiae. Studia in honorem Demetrii Protase, BistriţaCluj-Napoca, 2006, S. 483-489.

${ }^{80}$ Über diesen Gott vgl. J.R. Carbó Garcia, Los cultos orientales, S. 192.
} 
flamonii geschenkt, und er stellt ebenfalls eine gelungene Bestrebung zur Sozialbeförderung dar ${ }^{81}$.

Nur zwei Widmungen beziehen sich auf wirkliche exotische Götter, und zwar aus Palmyra. Es ist die Rede über die Erwähnung eines Tempels für die Dei Patrii einer palmyrenischen Sippe ${ }^{82}$ und über einen Altar für Deus Sol Ierhabol $^{83}$. Ihre Widmer sind tatsächlich palmyrenischer Abstammung, aber sie drücken sich in Latein aus. Insgesamt integrieren sich die Palmyrener sehr gut in die provinzielle Gesellschaft, obwohl sie ihre Traditionen bewahren ${ }^{84}$. Die Lage dieser Kulte in der Lokalgesellschaft kommt klar ans Licht durch die neuesten Entdeckungen in dem neben dem Forum liegenden palmyrenischen Tempel. Diese Kulte kommen in Mode und unter Caracalla bekommen sie offiziellen Status; aber in dem Kollegium ihrer cultores kommen weder spezifische Namen noch Mitglieder des ordo decurionum vor ${ }^{85}$.

Die Verehrung der orientalischen Götter bei den Dekurionen von Sarmizegetusa sieht ziemlich gering aus ${ }^{86}$. Wichtig ist auch die Tatsache, daß die Widmungen meistens aus persönlichen Gründen (ein einziges Mal ausdrücklich für die ganze Familie ${ }^{87}$ ) und nur zweimal für den Kaiser selbst ${ }^{88}$ erfolgen. Es ist abzuleiten, daß im Allgemeinen die uns zur Verfügung stehenden Texte eine aufrichtige persönliche Frömmigkeit ausdrücken.

\section{GOTTHEITEN AUS DEM ABENDLAND}

Diese Gottheiten erscheinen noch spärlicher. Wir können nur fünf vereinzelte Fälle anführen (7,69\%), für das religiöse Leben der Stadtaristokratie von geringer Bedeutung.

${ }^{81}$ CIL III 1134 (irrtümlicherweise nicht im IDR III/2 aufgenommen, vgl. IDR III/5, $2^{\star}$ ).

${ }^{82}$ CIL III 7954 = IDR III/2, 18; S. Nemeti, I Dii Patrii di Bene Agrud, [in:] L. Ruscu, C. Ciongradi, R. Ardevan, Cr. Roman, Cr. Găzdac (Hg.), Orbis antiquus. Studia in honorem Ioannis Pisonis, Cluj-Napoca 2004, S. 645-647. Es ist der einzige von einem Lokalaristokraten aufgestellte solcher Bau. Für den Tempel siehe A. Rusu-Pescaru, D. Alicu, Templele, S. 84-90.

${ }^{83}$ IDR III/1, 137 = AE 1977, 697.

${ }^{84}$ J.R. Carbó Garcia, Los cultos orientales, S. 245-251.

${ }^{85}$ I. Piso, O. Țentea, Un nouveau temple palmyrénien à Sarmizegetusa, Dacia. Revue d'archéologie et d'histoire ancienne. Nouvelle série 55, 2011, S. 118-121; iidem, Die palmyrenischen Truppen in Dakien. Monumente und Offentlichkeit, [in:] W. Eck, P. Funke, M. Dohnicht, K. Hallof, M. Heil, M. G. Schmidt (Hg.), Offentlichkeit - Monument - Text. XIV Congressus Internationalis Epigraphiae Graecae et Latinae 27.-31. Augusti MMXII. Akten, Berlin-Boston 2014, S. 480.

${ }^{86}$ J.R. Carbó Garcia, Los cultos orientales, S. 349.

${ }^{87}$ CIL III 7954 = IDR III/2, 18 (pro se suisque omnibus).

${ }^{88}$ IDR III/1, 137 = AE 1977, 697; CIL III 1100 = IDR III/5, 253. 
Eine Inschrift eines anonymen Widmers erwähnt die Obila, aber zusammen mit Herkules ${ }^{89}$; Iupiter Depulsor, eine Gottheit aus dem Ostalpenraum ${ }^{90}$, erscheint ebenfalls zusammen mit dem obersten römischen Gott ${ }^{91}$. Was die Widmung an Dis Pater und Proserpina anbelangt ${ }^{92}$, nur die bildliche Darstellung weist darauf hin, daß es sich um keltische Gottheiten handelt - Sucellus und Nantosuelta ${ }^{93}$. Auch die Quadriviae ${ }^{94}$, kleine Gottheiten der Kreuzwege, stammen aus demselben Kulturareal ${ }^{95}$, und der Widmer trägt auch einen keltischen Namen ${ }^{96}$.

Besondere Probleme gibt es im Zusammenhang mit dem Denkmal der rheinischen Göttinen Gesahenae ${ }^{97}$, von dem Ritter P. Aelius Maximus aus Napoca errichtet ${ }^{98}$. Auch wenn er zugleich in Sarmizegetusa Dekurione geworden ist, bleibt das vorliegende Stück wenig repräsentativ für die Stadt und drückt den persönlichen Geschmack dieses außerordentlichen Menschen aus ${ }^{99}$.

Wir können also behaupten, daß die Westgottheiten eine geringe Erscheinung in Sarmizegetusa darstellen ${ }^{100}$. Sie werden nur aus persönlichen Bedürfnissen verehrt und infolgedessen sind sie Gegenstand einer aufrichtigen Frömmigkeit.

${ }^{89}$ CIL III $6263=$ IDR III/4, 29. Üner diese einzigartige Gottheit siehe A. Husar, Celţi şi germani în Dacia romană, Cluj-Napoca 1999, S. 246.

${ }_{90}$ M. Šašel-Kos, Pre-Roman Divinities of the Eastern Alps and Adriatic, Ljubljana 1999, S. 125-129; S. Nemeti, I. Nemeti, Iupiter Depulsor în Dacia, Acta Musei Napocensis 39-40/I, 2002-2003 (2004), S. 242, 246.

${ }^{91}$ CIL III 12576 = IDR III/3, 17. Möglich ist auch ein Redigierungsfehler (die Wiederholung des Wortes Iovi); in diesem Fall wurde Depulsor als Epitheton von I.O.M. interpretiert.

${ }^{92}$ IDR III/2, 199.

${ }^{93}$ S. Nemeti, Cultul lui Sucellus - Dis Pater şi al Nantosueltei - Proserpina în Dacia romană, Ephemeris Napocensis 8, 1998, S. 101-110. Siehe auch A. Husar, Celţi şi germani, S. 249-254.

${ }^{94} \mathrm{IDR}$ III/5, 311.

${ }^{95}$ M. Ihm, Quadriviae, [in:] W.H. Roscher (Hg.), Ausführliches Lexikon der griechischen und römischen Mythologie, Leipzig IV, 1915, 5-7; A. Husar, Celţi şi germani, S. 246-249.

${ }^{96}$ IDR III/5, S. 248 (I. Piso).

${ }^{97}$ A. Husar, Celți şi germani, S. 241-242; I. Nemeti, Collective feminine goddesses in Roman Dacia, Acta Musei Napocensis 36/I, 1999, S. 149.

${ }^{98}$ AE 1969-1970, 548 = AE 1971, 395 = AE 1999, 1279 = ILD 544.

${ }_{99}$ M. Macrea, De la Burebista la Dacia postromană. Repere pentru o permanenţă istorică, ClujNapoca 1978, S. 134-135, 149-152; M. Bărbulescu, Interferențe, S. 213-214. Er widmet ein Denkmal einem weiteren rheinischen Gott, Hercules Magusanus (AE 1977, 702), aber dann war er noch keiner Mitglied des Adels von Sarmizegetusa (A. Szabó, Zur fragmentarischen Votivinschrift des P. Aelius Maximus sacerdos arae Augusti nostri aus Dazien, Acta Antiqua Academiae Scientiarum Hungaricae 39, 1999, S. 359-360).

${ }^{100}$ A. Husar, Celţi şi germani, S. 256. 


\section{UNBESTIMMTE GOTTHEITEN}

Es ist die Rede über sieben bruchstückhafte Inschriften, auf denen der Name der verehrten Gottheit nicht erhalten geblieben ist. Drei davon teilen uns nichts Weiteres mit $^{101}$. In zwei anderen Fällen besteht die gleiche Situation, nur daß der Name des Widmers teilweise erhalten ist ${ }^{102}$.

Die anderen zwei befinden sich in einer anderen Lage. Ziemlich gut erhalten ist die Basis einer Statue, die die Stadt zum Wohl des Kaisers Mark Aurel errichtet hat ${ }^{103}$; sie dokumentiert eine offiziele aber ehrliche und überzeugene Frömmigkeit ${ }^{104}$. Ein Altar wurde irgendwann zwischen 161-169 n. Chr. zur Gesundheit der Kaiser von einem Aristokraten aus dem Ritterstand aufgestellt ${ }^{105}$; seine Geste deutet auf eine politisch interessierte Tat.

\section{DER KAISERKULT}

Die Bedeutung dieses Kultes im öffentlichen Leben der Stadt liegt auf der Hand. Elf ulpiane Aristokraten bekleideten das Flaminat in der Stadt ${ }^{106}$, und andere fünf fungieten als sacerdotes arae Augusti ${ }^{107}$. Zu diesen kommen die vier schon erwähnten Widmungen der Kolonie zum Wohl und Erfolg des Kaisers hinzu ${ }^{108}$.

Die Beweise für ein persönliches Einsetzen des Lokaladels an diesem Kult sind aber äußerst gering. Die von diesen erbauten Denkmäler pro salute Augusti ${ }^{109}$ erscheinen in bloß drei Inschriften ${ }^{110}$. Dazu kommen noch die

\footnotetext{
${ }^{101}$ CIL III 7962 = IDR III/2, 353; CIL III 12585 = IDR III/2, 358; IDR III/3, 247.

${ }^{102}$ CIL III 1369 = IDR III/3, 149 (Lesung unsicher, vgl. R. Ardevan, Viaţa municipală, S. 396, Nr. 257); CIL III 7839 = IDR III/3, 335.

${ }^{103}$ I. Piso, Forum vetus, CEp 12.

${ }^{104}$ Während der Markomannenkriege wurde die Stadt angegriffen und bedroht (I. Piso, An der Nordgrenze, S. 61-66); die Rettung kam vom Kaiser (I. Piso, Forum vetus, S. 227).

${ }^{105}$ AE 1971,367 = IDR III/3, 245.

${ }^{106}$ R. Ardevan, Viaţa municipală, S. 136, 139, 525 (Tafel VII) .

${ }^{107}$ Ibidem, S. 332-333, 338-340, 610 (Tafel LVII, Nr. 4, 5, 6, 7, 9); für das letzte Stück siehe auch AE 1969-1970, $548=$ AE 1971, $395=$ AE 1999, $1279=$ ILD 544.

${ }^{108}$ CIL III 7958 + 7920 = IDR III/2, 227; IDR II/2, 241; CIL III 7986 = IDR III/2, 352; I. Piso, Forum vetus, CEp 12. Siehe oben, Anm. 19-20.

${ }^{109}$ Es wurden auch andere Formeln mit ähnlicher Bedeutung einbezogen.

${ }^{110}$ CIL III 1407 = IDR III/3, 10; AE 1971, 367 = IDR III/3, 245; CIL III 1100 = IDR III/5, 253.
} 
Erwähnungen über den Bau von zwei nymphaea in Sarmizegetusa, in honorem domus divinae ${ }^{111}$, von demselben Wohltäter stammend.

\section{FAZIT}

Das religiöse Leben der Mitglieder der Lokalaristokratie von Sarmizegetusa entfaltet sich in römischen Formen, in lateinischer Sprache und mit einer überwiegenden Vertretung der griechisch-römischen Kulte. Die orientalischen Einflüsse sind geringer als zu erwarten war, im Prozentsatz ausgedrückt sogar geringer als in anderen Städten der Provinz oder in anderen sozialen Milieus. Der Kaiserkult ist geringfügig in der Geistigkeit der Individuen.

Außerdem beweist die überwiegende Mehrheit der Votivtaten der ulpianischen Dekurionen eine aufrichtige, lebendige Frömmigkeit, weit entfern vom kalten Aussehen der offiziellen Zeremonien ${ }^{112}$. Die beliebtesten Gottheiten sind aber nicht die hochgestellten in dem offiziellen Pantheon (mit Ausnahme von Jupiter), sondern vor allem die, die den persönlichen Bedürfnissen der Gläubigen entgegenkommen ${ }^{113}$.

Schlussfolgernd kann gesagt werden, daß die Lokalaristokratie von Sarmizegetusa ein konservatives und konformistisches religiöses Verhalten römischen Typs aufweist. Dieses Verhalten ist ziemlich mannigfaltig und mit wenigen Einflüssen der fremden Religionen ${ }^{114}$. Aus diesem Standpunkt unterscheidet sich die Geistigkeit von Sarmizegetusa von der dynamischen und kosmopolitischen geistigen Tätigkeit in Apulum, oder von ihren reduzierten Formen anderer römisch-dakischen Städte.

${ }^{111}$ IDR III/2, 22 = AE 2003, 1520 und AE 2003, 1521 (vgl. I. Piso, Forum vetus, S. 244-248). Ebenfalls zur Ehre des Kaiserhauses wurde im Forum eine aedes fabrum mit dem Beitrag eines Stadtdekurionen erbaut (Ibidem, S. 227-230); das ist aber keine Votivgeste.

${ }^{112}$ M. Bărbulescu, Interferenţe, S. 229, 233-237.

${ }^{113}$ Ibidem, S. 250-258; M. Popescu, La religion, S. 329.

${ }^{114}$ R. Ardevan, Colonia Ulpia Traiana Sarmizegetusa, S. 280. 


\section{ON THE RELIGIOUS BEHAVIOR OF THE MUNICIPAL ARISTOCRACY FROM SARMIZEGETUSA}

\section{Summary}

The private votive inscriptions erected by the local aristocrats of Sarmizegetusa show their beliefs and religious preferences. One has first to choose carefully the sources, then to analyze them. The results are somehow surprising. The members of the local ordo decurionum are little involved personally in the imperial cult or in the exotic religions, either Oriental or Occidental. The most worshipped deities are, apart Jupiter, not the main Roman gods, but the ones defending the health, the home and the welfare of the humans. This aristocracy has a rather conservative religious behavior, but also a vivid and sincere faith, rather different from the cold official religion of the State.

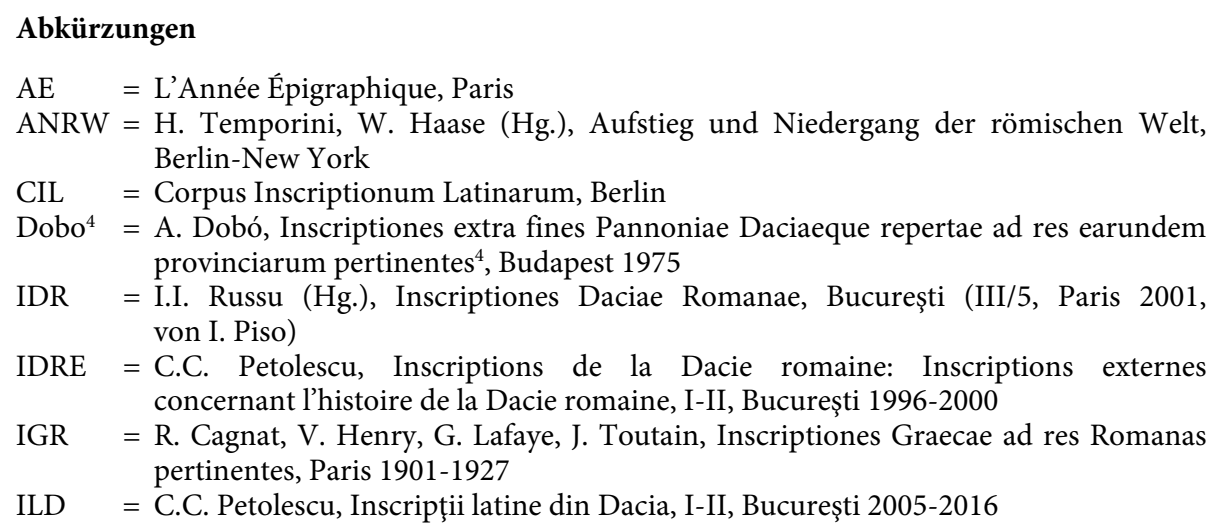

\section{Bibliographie}

Ardevan R., Viaţa municipală în Dacia romană, Timişoara 1998.

Ardevan R., Colonia Ulpia Traiana Sarmizegetusa: les institutions politiques et religieuses, [in:] I. Piso, R. Varga (Hg.), Trajan und seine Städte. Colloquium Cluj-Napoca, 29. September-2. Oktober 2013, Cluj-Napoca 2014, S. 275-285.

Bărbulescu M., Interferenţe spirituale în Dacia romană , Cluj-Napoca 2003.

Bruhl A., Liber Pater. Origine et expansion du culte dionysiaque à Rome et dans le monde romain, Paris 1953.

Carbó Garcia J.R., Los cultos orientales en la Dacia romana. Formas de diffusion, integración y control social e ideológico, Salamanca 2010.

Duthoy R., Les Augustales, [in:] Temporini H., Haase W. (Hg.), Aufstieg und Niedergang der Römischen Welt, II 16/2, Berlin-New York 1978, S. 1254-1309.

Dzino D., The cult of Silvanus: rethinking provincial identities in Roman Dalmatia, Vjesnik Arheološkog muzeja u Zagrebu 3, XLV, 2012, S. 261-279.

Etienne R., Piso I., Diaconescu Al., Les deux forums de la Colonia Ulpia Traiana Augusta Dacica Sarmizegetusa, Revue des Etudes Anciennes 92, 1990, 3-4, S. 273-296. 
Haensch R., Capita provinciarum. Statthaltersitze und Provinzialverwaltung in der römischen Kaiserzeit, Mainz 1993.

Husar A., Celţi şi germani în Dacia romană, Cluj-Napoca 1999.

Ihm M., Quadriviae, [in:] Roscher W.H., (Hg.), Ausführliches Lexikon der griechischen und römischen Mythologie IV, 1915, S. 1-7.

Macrea M., De la Burebista la Dacia postromană. Repere pentru o permanenţă istorică, ClujNapoca 1978.

Mócsy A., Pannonia and Upper Moesia. A History oft he Middle Danube Provinces of the Roman Empire, London-Boston 1974.

Nemeti I., Collective feminine goddesses in Roman Dacia, Acta Musei Napocensis 36/I, 1999, S. 135-153.

Nemeti S., Cultul lui Sucellus - Dis Pater şi al Nantosueltei - Proserpina în Dacia romană, Ephemeris Napocensis 8, 1998, S. 95-120.

Nemeti S., I Dii Patrii di Bene Agrud, [in:] Ruscu L., Ciongradi C., Ardevan R., Roman Cr., Găzdac Cr. (Hg.), Orbis antiquus. Studia in honorem Ioannis Pisonis, Cluj-Napoca 2004, S. 643-650.

Nemeti S., Nemeti I., Iupiter Depulsor în Dacia, Acta Musei Napocensis 39-40/I, 2002-2003 (2004), S. 241-246.

Nemeti S., Nemeti I., Theos Hypsistos epekoos la Sarmizegetusa, [in:] Gaiu, C., Găzdac, Cr. (Hg.), Fontes historiae. Studia in honorem Demetrii Protase, Bistriţa-Cluj-Napoca 2006, S. 483-489.

Noeske H.-Chr., Studien zur Verwaltung und Bevölkerung der dakischen Goldbergwerke in römischer Zeit, Bonner Jahrbücher des Rheinischen Landesmuseums 177, 1977, S. 373-426.

Pilipović S., Il culto di Liber a Taurunum; un contributo allo studio della viticoltura nella Pannonia Inferiore, Eirene. Studia Graeca et Latina 52, 2016, S. 445-470.

Piso I., Die soziale und ethnische Zusammensetzung der Bevölkerung in Sarmizegetusa und in Apulum, [in:] Eck W. (Hg.), Prosopographie und Sozialgeschichte. Studien zur Metodik und Erkentnismöglichkeit der kaiserzeitlichen Prosopographie (Kolloquium Köln, 24.-26. November 1991), Köln-Wien-Weimar 1993, S. 315-337.

Piso I., An der Nordgrenze des Römischen Reiches. Ausgewählte Studien (1972-2003), Stuttgart 2005.

Piso I. (Hg.), Le forum vetus de Sarmizegetusa, I, Bucureşti, 2006 (Corpus Epigraphicum, S. 211-332).

Piso I., Il Capitolium, l'Epulum Iovis e il Dies Iovis nella Dacia romana, [in:] Zerbini L. (Hg.), Roma e le province del Danubio. Atti del I Convegno Internazionale Ferrara - Cento, 15-17 Ottobre 2009, Soveria Manelli 2010, S. 269-278.

Piso I., Fasti provinciae Daciae II. Die ritterlichen Amtsträger, Bonn 2013.

Piso I., Țentea O., Un nouveau temple palmyrénien à Sarmizegetusa, Dacia. Revue d'archéologie et d'histoire ancienne. Nouvelle série 55, 2011, S. 111-121.

Piso I., Marcu F., Cupcea G., Țentea O., Varga R., Das Kapitol von Sarmizegetusa, Dacia. Revue d'archéologie et d'histoire ancienne. Nouvelle série 56, 2012, S. 119-123.

Piso I., Ţentea O., Die palmyrenischen Truppen in Dakien. Monumente und Offentlichkeit, [in:] Eck W., Funke, P., Dohnicht M., Hallof K., Heil M., Schmidt M.G. (Hg.), Offentlichkeit - Monument - Text. XIV Congressus Internationalis Epigraphiae Graecae et Latinae 27.-31. Augusti MMXII. Akten, Berlin-Boston 2014, S. 479-480.

Popescu C., Popescu, M., Le culte de Liber Pater en Dacie romaine, Thraco-Dacica 16, 1995, S. 227-235.

Popescu M., La religion de l'armée romaine en Dacie, Bucarest 2002.

Rusu-Pescaru A., Alicu D., Templele romane din Dacia, I, Deva 2000.

Sanie S., Cultele orientale în Dacia romană. I Cultele siriene şi palmiriene, Bucureşti 1981.

Šašel-Kos M., Pre-Roman Divinities of the Eastern Alps and Adriatic, Ljubljana 1999.

Szabó A., Zur fragmentarischen Votivinschrift des P. Aelius Maximus sacerdos arae Augusti nostri aus Dazien, Acta Antiqua Academiae Scientiarum Hungaricae 39, 1999, S. 355-361. 
